\title{
Time series statistical analysis: a powerful tool to evaluate the variability of resistive switching memories
}

\author{
J.B. Roldán ${ }^{1 *}$, F. J. Alonso², A. M. Aguilera ${ }^{2}$, D. Maldonado ${ }^{1}$, M. Lanza ${ }^{3}$ \\ ${ }^{1}$ Departamento de Electrónica y Tecnología de Computadores. Universidad de Granada. \\ Facultad de Ciencias. Avd. Fuentenueva s/n, 18071 GRANADA, Spain. \\ *Corresponding author Email: jroldan@ugr.es \\ ${ }^{2}$ Departamento de Estadística e Investigación Operativa. Universidad de Granada. \\ Facultad de Ciencias. Avd. Fuentenueva s/n, 18071 GRANADA, Spain. \\ ${ }^{3}$ Institute of Functional Nano and Soft Materials (FUNSOM), Collaborative Innovation \\ Center of Suzhou Nanoscience \& Technology, Soochow University, 199 Ren-Ai Road, \\ Suzhou, 215123, China.
}

\section{ABSTRACT}

Time series statistical analyses (TSSA) have been employed to evaluate the variability of resistive switching memories, and to model the set and reset voltages for modeling purposes. The conventional procedures behind time series theory have been used to obtain autocorrelation and partial autocorrelation functions and determine the simplest analytical models to forecast the set and reset voltages in long series of resistive switching processes. To do so, and for the sake of generality in our study, a wide range of devices have been fabricated and measured. Different oxides and electrodes have been employed, including bilayer dielectrics in devices such as: 
$\mathrm{Ni} / \mathrm{HfO}_{2} / \mathrm{Si}-\mathrm{n}^{+}, \mathrm{Cu} / \mathrm{HfO}_{2} / \mathrm{Si}-\mathrm{n}^{+}$and $\mathrm{Au} / \mathrm{Ti} / \mathrm{TiO}_{2} / \mathrm{SiO}_{\mathrm{x}} / \mathrm{Si}^{-} \mathrm{n}^{+}$. The TSSA models obtained allowed to forecast the reset and set voltages in a series if previous values were known. The study of autocorrelation data between different cycles in the series allows estimating the inertia between cycles in long resistive switching series. Overall, TSSA seems to be a very promising method to evaluate the intrinsic variability of resistive switching memories.

Index Terms - Resistive switching memory, RRAM, Conductive filaments, Variability, Time series modelling, Autocovariance, Stationary time series.

\section{1 - INTRODUCTION}

Resistive random access memories (RRAM) have shown an outstanding potential for information storage, especially for internet of things (IoT) and related applications, due to their excellent scalability, low power operation, fast switching speed, easy fabrication and good compatibility with the well-established complementary metal-oxide-semiconductor (CMOS) technology [1, 2, 3, 4, 5, 6, 7]. The most promising RRAM unit cells consist of matrixes of two-terminal metal/insulator/metal (MIM) nanocells, in which the electrical resistance of the insulating film can be switched between a high resistive state (HRS) and a low resistive state (LRS) depending on the electrical impulses applied between the two metallic electrodes. However, after more than one decade of intense research, RRAM devices still have not been mass produced by the industry, nor entered in the market of non-volatile memories (NVM), mainly due to variability problems $[1,2,5,8,9,10]$. In RRAM devices the resistive switching (RS) 
is related to the formation and rupture of defect-rich conductive filaments (CFs) within the dielectric layer, which leads to a HRS-to-LRS (set) and a LRS-to-HRS (reset) transition (respectively). Set and reset transitions are related to diffusion, redox and nucleation of different chemical species within the MIM nanocells $[1,2,5,7,10]$, which take place with a very high degree of randomness from one cycle to another, leading to an intrinsic high variability. Consequently, the electrical characteristics measured in a RRAM device reflects the stochasticity of these physical processes, and produces the so-called cycle-to-cycle variability.

In the past few years, the variability of RRAM devices has been statistically analysed in most cases using the Weibull distribution (WD) $[1,11,12]$. The WD comes out in the field of reliability physics [13] and its use makes sense for RRAMs under filamentary conduction since it is a weakest-link type distribution, i.e. the failure of the whole is dominated by the degradation rate for the weakest element. However, this method does not describe all the inherent statistical particularities of RRAM devices: and although it has been previously employed to deepen on the experimental data characteristic obtained for different technologies $[1,11,12]$, it does not entirely capture the essence of the RS process. In fact, classical reliability analyses with the Weibull distribution assume that times to failure, in our case set voltage $\left(V_{S E T}\right)$ and/or reset voltage $\left(V_{R E S E T}\right)$, are independent within a RS series. This assumption may not be valid in the case of stochastic processes associated with RRAMs because successive observations could be highly dependent (in fact, a CF is formed making use of broken parts of previous ones). It is worth highlighting that other mathematical approaches have been proposed to tackle different facets of the statistical study of variability in RRAMs and, in a more general scope, thin dielectrics. Among them, the use of a clustering statistical approach complementing the use of the WD [14] can be counted; in 
line with this, convolution-based modelling is also interesting being noticed [15]. Strategies making use of completely different distribution functions have also been reported; for example, the employment of phase-type distribution functions for certain devices led to interesting results [16]. Markov models have also been employed in the analysis of these devices $[17,18]$. Kinetic Monte Carlo (KMC) and related simulations can also be considered as statistical tools to analyse RRAM variability, as they allow modifying very specific physical parameters of the devices (i.e. concentration of atomic vacancies/dopants, insulator thickness fluctuations) within a reasonable range and analyse the deviation of the electrical characteristics. A broad number of contributions have been presented in this respect [10, 19-25]. The only drawback of KMC simulations is the longer computational time, which obligates researchers to make assumptions that simplify the calculations, leading in some cases to a loss of accuracy.

It is clear that variability is still an unresolved problem in RRAM devices both from the technological and the modelling viewpoint, and developing new analytical methods to shed light into this problem is highly necessary. Times series statistical analyses (TSSA) are powerful numerical methods that have been successfully applied for decades in the fields of economics and sociology, and more recently they have been also sporadically used in the field of engineering and reliability of electronic devices [26-27]. TSSA may be useful to analyse the variability of RRAM devices because: i) the data $\left(V_{S E T}\right.$ and $\left.V_{R E S E T}\right)$ are collected in a continuous manner over the time (cycle-tocycle) for a long RS series [28-29]; ii) TSSA is appropriate for physical processes that exhibit any kind of inertia in some of their particular features [30, 31]. In this respect, in RS cycling, the CF is formed (set process) making use of the remnants of the CFs ruptured in the previous cycle (reset process). Therefore, from a statistical point of view it is relevant to analyse any numerical relations between neighbouring cycles and assess 
the system "memory" in a long RS series (to put it in formal words, the parameters that characterize consecutive cycles in a RS series can be correlated and, therefore, the term autocorrelation comes up naturally). And iii) under certain mathematical conditions (i.e. time series stationarity, an assumption that our data distributions fulfil) a comprehensive analysis can be performed through a time series analysis approach [30, 31]. However, despite this strong parallelism, to the best of our knowledge, TSSA have never been employed to evaluate the variability of RRAM devices. In this work we present the first variability study of three different types of RRAM devices using the TSSA, and observe that in all cases essential RS parameters, such as $V_{S E T}$ and $V_{R E S E T}$, can be reasonably forecasted making use of mathematical models and the information of these parameters in previous cycles. In addition, in long RS series the dependence of forthcoming cycles on previous ones can be correctly studied by means of correlation and autocorrelation analyses, characterizing in this manner the inertia of RS operation in RRAMs for different technologies.

The manuscript is organized as follows: in Section II the new model is described in depth, in Section III the fabricated devices and measurement process details are given, in Section IV the new statistical analysis is explained, and in Section V the main results and discussion are presented. Finally, the main conclusions are drawn in Section VI. 


\section{2 - MODEL DESCRIPTION}

Back in 1927, G.U. Yule introduced modern TSSA formulating a model for a pendulum dynamic movement time dependency [32]. After rearrangements in the corresponding equation describing the pendulum movement he came out with a second order autoregressive time series model where the pendulum displacement $\left(z_{t}\right)$ from the equilibrium position was regressed on the two previous observations $\left(z_{t-1}\right)$ and $\left(z_{t-2}\right)-$ the physics governing the pendulum is linked to a second order differential equation-. In our study, we model the values of $V_{S E T}$ and $V_{R E S E T}$ of different RRAM devices over long series of RS cycles by considering the values of previous cycles using TSSA [30, $31,33]$. One of the main difficulties is to find the order of the model, i.e. how many $\mathrm{V}_{\text {SET }}$ or $\mathrm{V}_{\text {RESET }}$ values from previous cycles we need to forecast the current cycle (for a general model previous cycles are usually considered, see Equation 1). In addition, it is also necessary to find the weights $\left(\Phi_{1} \ldots \Phi_{p}\right)$ of the autoregressive model we are seeking (see Equation 1).

$$
V_{\text {reset }_{t}}=\Phi_{1} V_{\text {reset }_{t-1}}+\Phi_{2} V_{\text {reset }_{t-2}}+\cdots+\Phi_{p} V_{\text {reset }_{t-p}}+\varepsilon_{t}
$$

The order of the model $(p)$ depends on the physics governing RS, but we will not assume any knowledge of it and we will only make use of the information within the experimental data because the underlying physics and the technological details of the fabrication process are "hidden" in the RS data collected. Therefore, the models obtained with this approach are empirical and the TSSA output will consist of the order of the model $(p)$ and the weights set $\left(\Phi_{1}, \ldots, \Phi_{p}\right)$. Sometimes a model such as the one 
described in Equation 1 works better for the centred variables; e.g., $V_{R E S E T}-\mu$, where $\mu$ stands for $V_{R E S E T}$ mean in the RS series (this formulation is equivalent to include a constant term $\Phi_{0}$ in the model). The term $\varepsilon_{t}$, as usually employed in time series studies, stands for a residual that accounts for the model error (the difference between the measured value and the modelled value). This term is obtained in TSSA theory by generating random numbers with a normal distribution whose variance corresponds to the one calculated from the measured data. Nevertheless, for the sake of simplicity, this term will not be included in the models developed here, following the conventional notation in the engineering context. In this approach, the algebraic equations are employed in the form usually seen in compact modelling, i.e., the current value of reset or set voltages are given as a function of variables already known, such as reset and set voltages of previous cycles. The existence of a difference between the measured and modelled values is assumed. Equation 1 shows what is called an autoregressive (AR) model [30]; however, not always such an easy model can be obtained. Occasionally more complex models are needed; if this is the case, an autoregressive moving average (ARMA) model that includes AR and moving average (MA) parts are considered [30]. MA models are a linear combination of past residuals [30-32]. The general expression of an ARMA model is described in the Supplementary Information (see Equation A1).

We have employed TSSA to study and model the data obtained from RRAMs made of different materials. Three types of devices were considered, two of them including $\mathrm{HfO}_{2}$ as the dielectric, sandwiched by different electrode materials ( $\mathrm{Ni}$ and $\mathrm{Cu})$, and another one based on a bilayer $\left(\mathrm{TiO}_{2} / \mathrm{SiO}_{\mathrm{X}}\right)$ insulating stack. 


\section{3 - DEVICE FABRICATION AND MEASUREMENT}

The $\mathrm{HfO}_{2}$-based RRAM devices consisted of $\mathrm{Ni} / \mathrm{HfO}_{2} / \mathrm{Si}-\mathrm{n}^{+}$and $\mathrm{Cu} / \mathrm{HfO}_{2} / \mathrm{Si}-\mathrm{n}^{+}$ (20nm thick dielectric layers were used) stacks [28]. The active area of the MIM cells was $5 \mu \mathrm{m} \times 5 \mu \mathrm{m}$, and the devices were patterned by photolithography. A HP-4155B semiconductor parameter analyser was used in the measurement process, which consisted of collecting long sequences (series) of current vs. voltage (I-V) curves by applying ramped voltage stress (RVS). The $\mathrm{Si}^{+} \mathrm{n}^{+}$substrate (bottom) electrode was grounded and a negative voltage was applied to the $\mathrm{Ni}(0.3 \mathrm{~V} / \mathrm{s}$ ramps $)$ or $\mathrm{Cu}(0.5 \mathrm{~V} / \mathrm{s}$ ramps) (top) electrode, although for simplicity we have assumed the absolute value of the applied voltage henceforth [28]. The RS phenomenon observed for both type of devices was unipolar, as displayed by the I-V curves (see Figures 1a, 1b) [5].

A RS series of 2800 cycles was obtained for the $\mathrm{Ni} / \mathrm{HfO}_{2} / \mathrm{Si}-\mathrm{n}^{+} \mathrm{RRAMs}$, and a series of 280 cycles for the $\mathrm{Cu} / \mathrm{HfO}_{2} / \mathrm{Si}-\mathrm{n}^{+}$RRAMs. The values of $V_{S E T}$ and $V_{R E S E T}$ were extracted from the I-V curves as reported in [1, 28, 34], and plotted in Figures 1b-1f. The variability of $V_{S E T}$ and $V_{R E S E T}$ from one cycle to another can be clearly observed. 

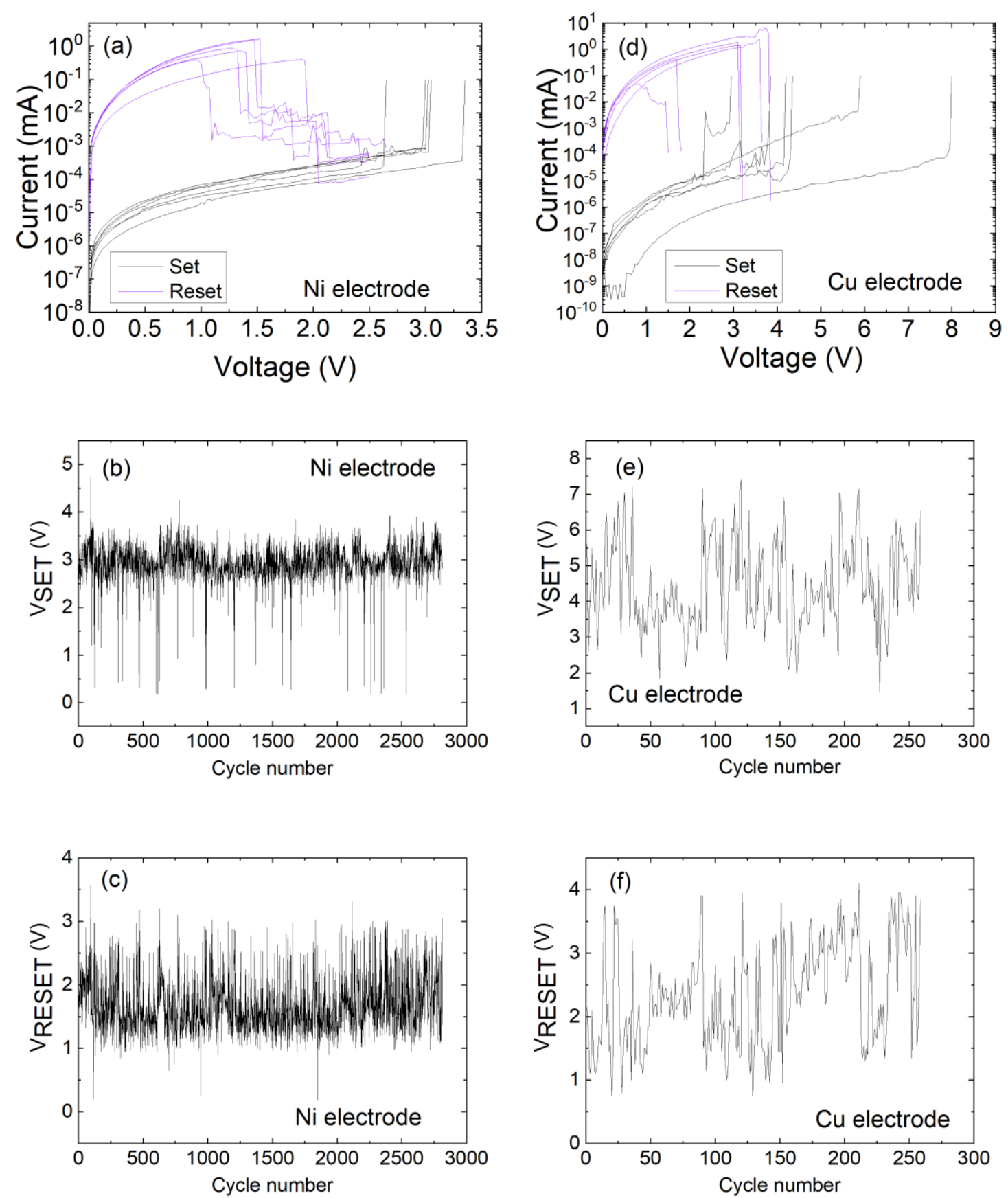

Figure 1. Typical I-V curves observed in (a) $\mathrm{Ni} / \mathrm{HfO}_{2} / \mathrm{Si}-\mathrm{n}^{+}$and (d) $\mathrm{Cu} / \mathrm{HfO}_{2} / \mathrm{Si}-\mathrm{n}^{+}$ RRAMs. Experimental values of $V_{S E T}(\mathrm{~b}, \mathrm{e})$ and $V_{R E S E T}(\mathrm{c}, \mathrm{f})$ versus cycle number for a series of continuous RS cycles under RVS for RRAMs based on $\mathrm{Ni} / \mathrm{HfO}_{2} / \mathrm{Si}-\mathrm{n}^{+}$and $\mathrm{Cu} / \mathrm{HfO}_{2} / \mathrm{Si}-\mathrm{n}^{+}$stacks.

The third type of RRAM devices was fabricated using an $\mathrm{Au} / \mathrm{Ti} / \mathrm{TiO}_{2} / \mathrm{SiO}_{\mathrm{X}} / \mathrm{Si}-\mathrm{n}^{+}$ structure. A $2 \mathrm{~nm} \mathrm{TiO}_{2}$ film was grown by atomic layer deposition (ALD), on $\mathrm{Si}-\mathrm{n}^{+}$ wafer with a $\sim 1.5 \mathrm{~nm}$ thick native $\mathrm{SiO}_{\mathrm{x}}$ layer. The device area was $5 \mu \mathrm{m}$ x $5 \mu \mathrm{m}$ and the 
thicknesses of other layers were $\mathrm{Au}(60 \mathrm{~nm})$ and $\mathrm{Ti}(20 \mathrm{~nm})$. The Ti interfacial top electrode was employed as a gathering layer for oxygen accumulation. More details about the fabrication process of these structures are given in Ref. [29]. 0.5V/s ramps were employed for the series measured in this case.
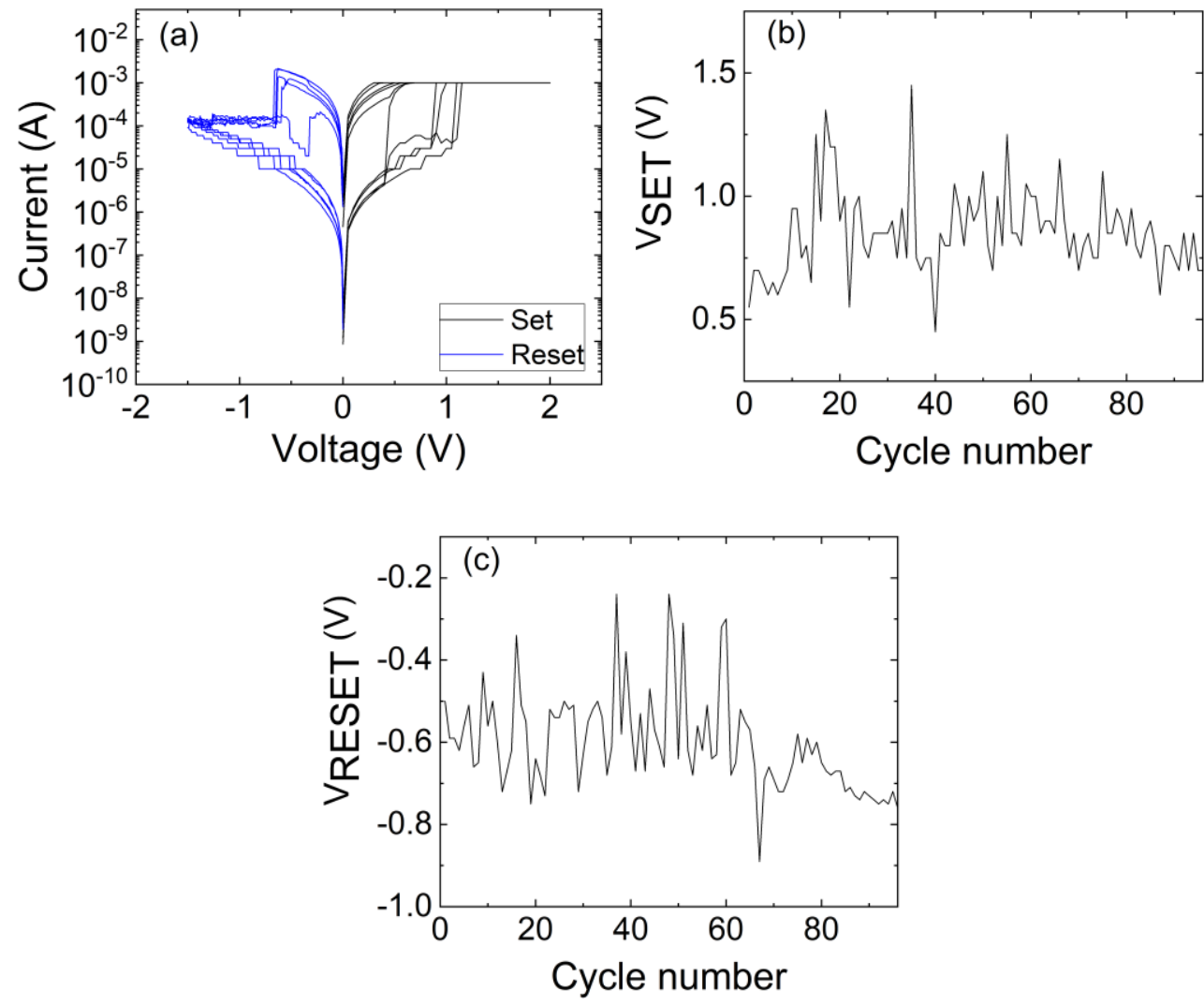

Figure 2. (a) Typical $\mathrm{I}-\mathrm{V}$ curves observed in $\mathrm{Au} / \mathrm{Ti} / \mathrm{TiO}_{2} / \mathrm{SiO}_{\mathrm{X}} / \mathrm{Si}-\mathrm{n}^{+} \mathrm{RRAMs}$. Experimental values of $V_{S E T}$ (b) and $V_{R E S E T}$ (c) versus cycle number for a series of continuous resistive switching cycles under RVS.

Some of the $\mathrm{I}-\mathrm{V}$ curves for the $\mathrm{Au} / \mathrm{Ti} / \mathrm{TiO}{ }_{2} / \mathrm{SiO} / \mathrm{Si}-\mathrm{n}^{+}$devices are plotted in Figure 2, which shows clear bipolar RS behaviour, and the extracted values of the set and reset voltages for a RS series of 100 cycles. As it can be seen, the different type of RS (bipolar) compared to Figure 1 (unipolar) also leads to different patterns in the $V_{S E T}$ and $V_{\text {RESET }}$ plots. For the devices based on $\mathrm{HfO}_{2}$ dielectrics the set (reset) voltage were 
determined by detecting a $70 \%$ current increase (decrease) with respect to the previous current point. For the devices with the $\mathrm{TiO}_{2}$ dielectric the current maximum was selected to determine the reset voltage and the maximum change in the derivative was the choice for the determination of the set voltage. More details on these methods are given in Ref. [34].

\section{4 - NEW STATISTICAL METHODOLOGY}

We have employed TSSA to analyze the experimental $V_{S E T}$ and $V_{R E S E T}$ plotted in Figure 1 and Figure 2 for the technologies under consideration. A detailed description of this statistical methodology is given in the Supplementary Information. This new statistical approach for RRAM variability modeling was implemented with the packages TSA and forecast in R language [35].

The first step of our methodology is to prove that our data constitute a stationary series $[30,31,33]$. This implies that the mean and variance are constant in time (RS cycle). Figure 1 indicates that the values are distributed around the mean value and no pattern in the data fluctuation can be found. This fact suggests that both the data mean and variance are constant all along the series, i.e. for all the cycle intervals considered - a numerical check of these issues has been also performed-. Therefore, according to the explanation given in the Supplementary Information, all the data series under consideration for the devices $\mathrm{Ni} / \mathrm{HfO}_{2} / \mathrm{Si}-\mathrm{n}^{+}$and $\mathrm{Cu} / \mathrm{HfO}_{2} / \mathrm{Si}-\mathrm{n}^{+}$are stationary. For the $\mathrm{Au} / \mathrm{Ti} / \mathrm{TiO}_{2} / \mathrm{SiO}_{\mathrm{x}} / \mathrm{Si}-\mathrm{n}^{+}$devices we obtain similar results in what is related to the stationarity of the series, i.e. the data are distributed around the mean value and no data fluctuation patterns are seen. 
The second step is to select the most parsimonious ARMA model to forecast the voltage to reset/set in one cycle in terms of the voltages to reset/set in previous cycles. A parsimonious model is the simplest model (algebraically speaking) that can be used to correctly model a certain phenomenon [31].

\section{1 - Modeling $V_{S E T}$}

For the $V_{S E T}$ distributions considered in this investigation, the mean values are $\hat{\mu}_{\mathrm{VSET}}=2.934 \mathrm{~V}\left(\mathrm{Ni} / \mathrm{HfO}_{2} / \mathrm{Si}-\mathrm{n}^{+}\right.$devices $)$and $\hat{\mu}_{\mathrm{VSET}}=4.433 \mathrm{~V}\left(\mathrm{Cu} / \mathrm{HfO}_{2} / \mathrm{Si}^{-\mathrm{n}^{+}}\right.$devices $)$. The autocorrelation functions (ACFs) and partial autocorrelation functions (PACFs) of the data samples are shown in Figure 3. The ACF is a function of the number of cycles $k$ and measures the influence/connection between $V_{S E T} / V_{R E S E T}$ separated by $k$ cycles $(k$ distant lags). On the other hand, the PACF measures the same correlation but eliminating the dependency due to the intermediated lags $(1,2, \ldots, k-1)$. That is, in the PACF the dependencies of each two cycles are evaluated making sure that statistical crossed dependencies by means of cycles in between are eliminated. A simple example to illustrate this concept can be built with a 3 elements series. For this series, the calculation of PACF between the first and third elements would need the elimination of the dependencies provided by the second on the first and the second on the third elements; in this way, the direct dependencies of the first on the third could be calculated. 

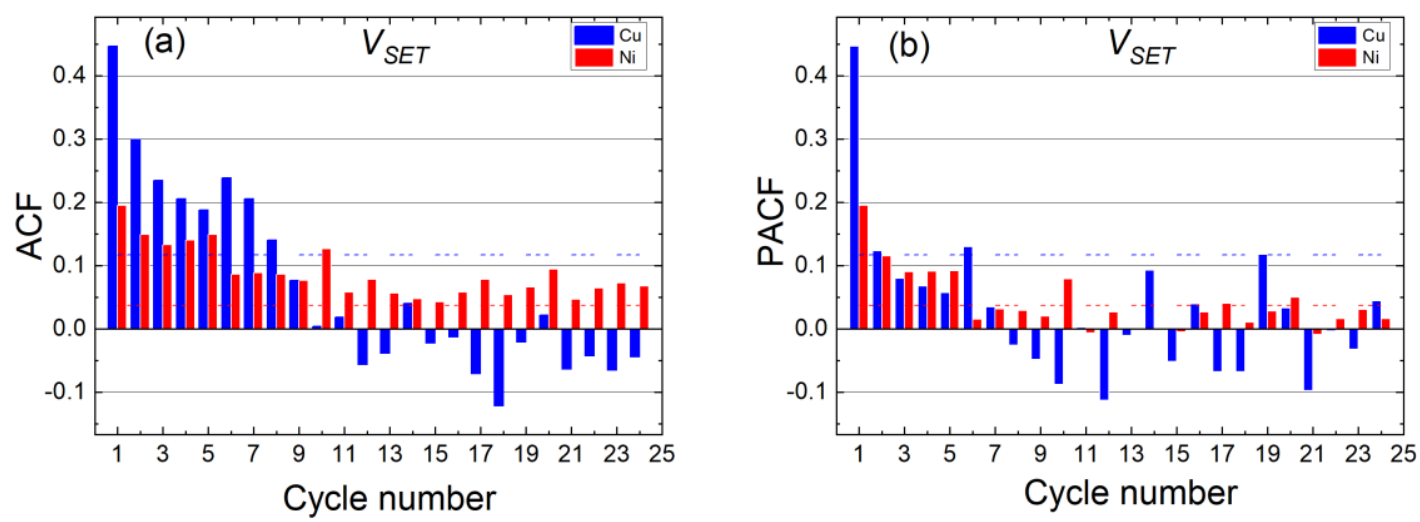

Figure 3. (a) ACF and (b) PACF versus cycle lag (distance apart in cycles within a RS series; for a cycle lag 1 the ACF and PACF of consecutive cycles are measured and so on) for the $V_{S E T}$ series described in Figure 1 , corresponding to $\mathrm{Ni} / \mathrm{HfO}_{2} / \mathrm{Si}-\mathrm{n}^{+}$and $\mathrm{Cu} / \mathrm{HfO}_{2} / \mathrm{Si}^{+} \mathrm{n}^{+}$devices. The ACF and PACF minimum threshold bounds for the $\mathrm{Cu} / \mathrm{HfO}_{2} / \mathrm{Si}-\mathrm{n}^{+}$and $\mathrm{Ni} / \mathrm{HfO}_{2} / \mathrm{Si}-\mathrm{n}^{+}$devices are 0.117 and 0.037 respectively, shown with dashed lines.

As Figure 3a shows, the $\mathrm{ACF}$ for $\mathrm{Cu} / \mathrm{HfO}_{2} / \mathrm{Si}-\mathrm{n}^{+}$devices is higher than for $\mathrm{Ni} / \mathrm{HfO}_{2} / \mathrm{Si}-\mathrm{n}^{+}$devices for the first two lag cycles (see that the blue columns are higher). In fact, we have just one component, the corresponding to the previous cycle, that dominates over the rest. These values have to be compared with the threshold bounds (Equation A10 in the Supplementary Information for ACF, which depends on the number of cycles of the series). In the PACF plot shown in Figure $3 b$ (see also Equation A9 in the Supplementary Information), only the first value is above the threshold bound. Consequently, following the procedure to select the simplest model depicted in the Supplementary Information (Model identification section), an AR (1) model is proposed for the $\mathrm{Cu} / \mathrm{HfO}_{2} / \mathrm{Si}-\mathrm{n}^{+}$devices (ACF decreases and PACF has only one significant value). This can be translated to Equation 2, that reflects that the $V_{S E T}$ model of a current cycle just depends on the value of the previous cycle:

$$
V_{S E T_{t}}(V)=2.4263+0.4527 V_{S E T_{t-1}}
$$

The numbers included in Equation 2 can be obtained as described in the Supplementary Information (Parameter estimation section). In this case and henceforth, 
as explained in the introduction, we will not include the residual for the current cycle $\left(\varepsilon_{t}\right)$, as usually done in a mathematician context. For $\mathrm{Ni} / \mathrm{HfO}_{2} / \mathrm{Si}-\mathrm{n}^{+}$devices, the $\mathrm{ACF}$ and PACF have several values outside the corresponding thresholds (the minimum threshold bounds for the $\mathrm{Cu} / \mathrm{HfO}_{2} / \mathrm{Si}-\mathrm{n}^{+}$and $\mathrm{Ni} / \mathrm{HfO}_{2} / \mathrm{Si}-\mathrm{n}^{+}$devices are 0.117 and 0.037 respectively) and both functions decrease. The model selection procedure suggests an ARMA $(1,1)$ model. Thus, $V_{S E T}$ for the $\mathrm{Ni} / \mathrm{HfO}_{2} / \mathrm{Si}-\mathrm{n}^{+}$RRAMs can be described by means of Equation 3:

$$
V_{S E T_{t}}(V)=0.2380+0.9189 V_{S E T_{t-1}}+0.8049 \varepsilon_{t-1} \text {. }
$$

As it can be seen, the structure of the autocorrelation relations between cycles for the set voltage series lead to a more complex model for the $\mathrm{Ni} / \mathrm{HfO}_{2} / \mathrm{Si}-\mathrm{n}^{+}$devices. Finally, the residuals (i.e. the difference between the measured and modeled values for each cycle) of both models (Equations 2 and 3) were computed and the white noise behavior (uncorrelated errors) was satisfactorily checked, as it is usually done.

For the $\mathrm{Au} / \mathrm{Ti} / \mathrm{TiO}_{2} / \mathrm{SiO}_{\mathrm{x}} / \mathrm{Si}-\mathrm{n}^{+}$devices the $\mathrm{ACF}$ and PACF have several values outside the corresponding thresholds and both functions decrease.
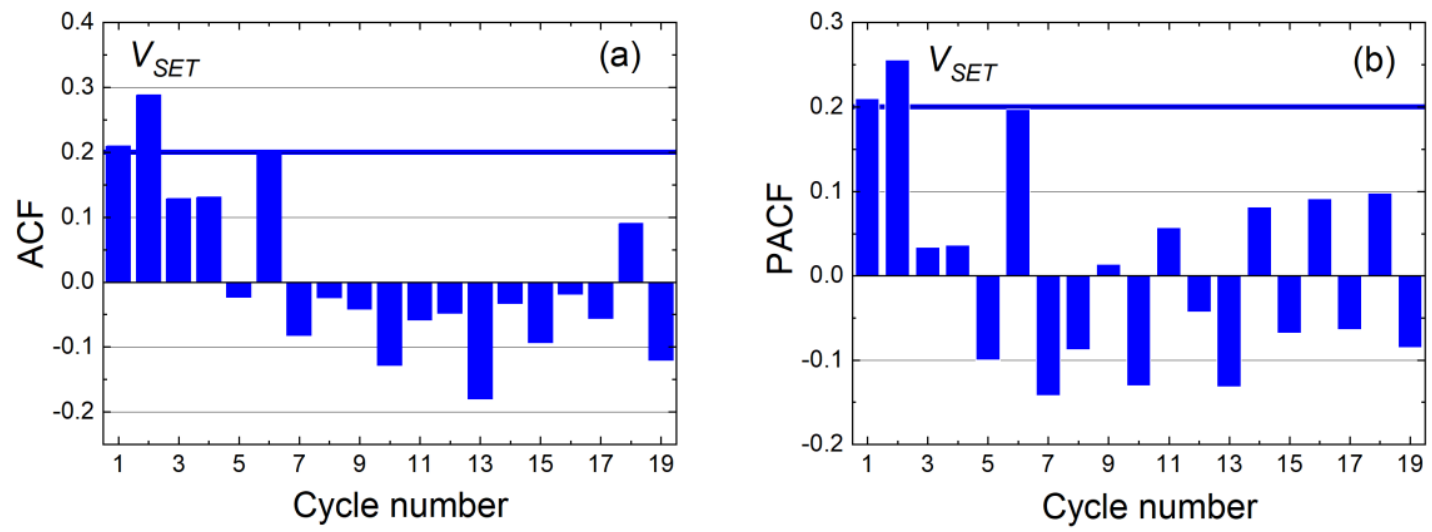

Figure 4. (a) ACF and (b) PACF versus cycle lag (distance apart in cycles within a RS series; for a cycle lag 1 the ACF and PACF of consecutive cycles are measured and so on) for the $V_{\text {SET }}$ series described in Figure 2, corresponding to $\mathrm{Au} / \mathrm{Ti} / \mathrm{TiO}_{2} / \mathrm{SiO}_{\mathrm{x}} / \mathrm{Si}-\mathrm{n}^{+}$ devices. The ACF and PACF minimum threshold bounds is 0.2 , shown in solid line. 
The model selection procedure suggests an AR (2) model with a null coefficient for the previous cycle component $\left(V_{S E T(t-1)}\right)$. Therefore, for this case $V_{S E T}$ is given in Equation 4:

$$
V_{S E T_{t}}(V)=0.6051+0.2926 V_{S E T_{t-2}}(V)
$$

\section{2 - Modeling $V_{R E S E T}$}

For the $V_{R E S E T}$ model we have proceeded in a similar manner. The mean values are the following: $\hat{\mu}$ VRESET $=2.358 \mathrm{~V}\left(\mathrm{Cu} / \mathrm{HfO}_{2} / \mathrm{Si}^{-} \mathrm{n}^{+}\right)$and $\hat{\mu}$ VRESET $=1.665 \mathrm{~V}\left(\mathrm{Ni} / \mathrm{HfO}_{2} / \mathrm{Si}-\right.$ $\mathrm{n}^{+}$). The sample ACF for the measured series of Figure 1 are given in Figure 5a, and the sample PACF is given in Figure 5b. The greater height of the blue bars in Figure 5, both in the ACF and PACF, indicate a higher autocorrelation function for $\mathrm{Cu} / \mathrm{HfO}_{2} / \mathrm{Si}-\mathrm{n}^{+}$ devices in comparison to $\mathrm{Ni} / \mathrm{HfO}_{2} / \mathrm{Si}-\mathrm{n}^{+}$devices.
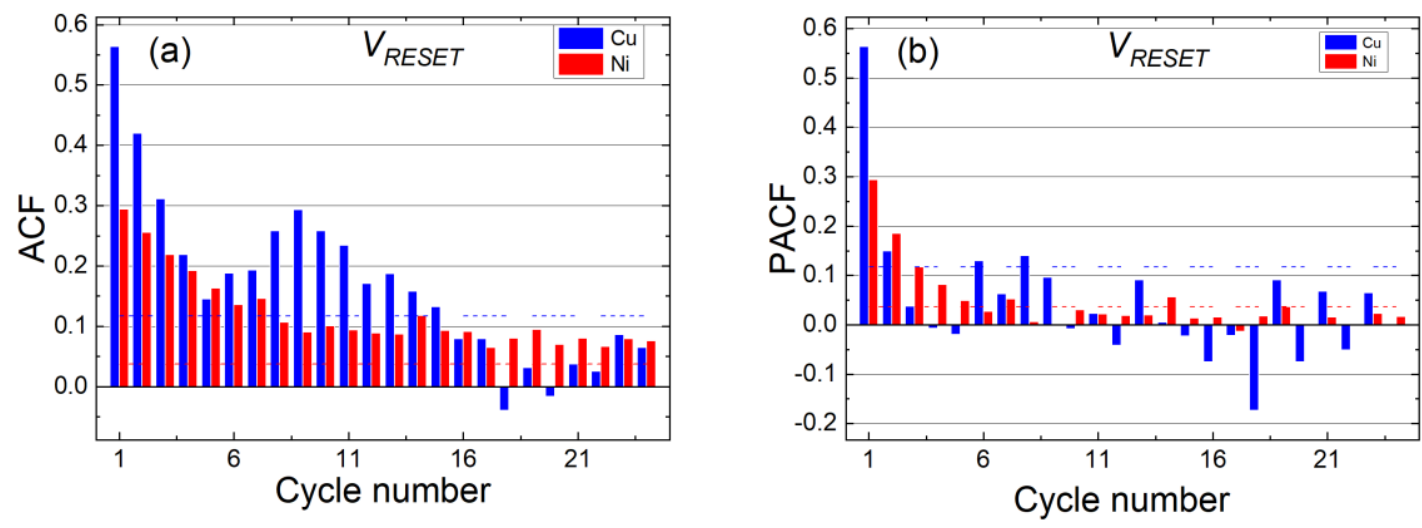

Figure 5. (a) ACF and (b) PACF versus cycle lag for the reset voltage of the $\mathrm{Cu} / \mathrm{HfO}_{2} / \mathrm{Si}-\mathrm{n}^{+}$and $\mathrm{Ni} / \mathrm{HfO}_{2} / \mathrm{Si}-\mathrm{n}^{+}$devices under study and the $\mathrm{RS}$ series described in section II. The ACF minimum threshold bounds for the $\mathrm{Cu} / \mathrm{HfO}_{2} / \mathrm{Si}-\mathrm{n}^{+}$and $\mathrm{Ni} / \mathrm{HfO}_{2} / \mathrm{Si}$ $\mathrm{n}^{+}$devices are 0.117 and 0.037 respectively, shown with dashed lines. 
See that the sample PACF for $\mathrm{Cu} / \mathrm{HfO}_{2} / \mathrm{Si}^{+} \mathrm{n}^{+} \mathrm{RRAMs}$ has only two values (the first and second lagged cycles) above the threshold bound (Equation A9), so that an AR (2) model could be considered. However, taking into consideration that the second component is close to the threshold bound an AR (1) can be reasonable. After the AR (1) is adjusted all the validation considerations are satisfied. The $V_{R E S E T}$ model for these devices is given in Equation 5:

$$
V_{R E S E T_{t}}(V)=0.8577+0.5711 V_{R E S E T_{t-1}} .
$$

For the $\mathrm{Ni} / \mathrm{HfO}_{2} / \mathrm{Si}-\mathrm{n}^{+}$devices, the $V_{\text {RESET }}$ model works similarly to what was determined for the set voltage modeling (ACF and PACF decrease). Then an ARMA $(1,1)$ model (Equation 5) holds for the $V_{\text {RESET }}$ time series for $\mathrm{Ni} / \mathrm{HfO}_{2} / \mathrm{Si}^{-} \mathrm{n}^{+}$devices:

$$
V_{R E S E T_{t}}(V)=0.2377+0.8573 V_{R E S E T_{t-1}}+0.6523 \varepsilon_{t-1} .
$$

The residuals of the $V_{R E S E T}$ models depicted in Equations 5 and 6 have white noise behavior again, and confirm the appropriateness of the modeling procedure.

For the $\mathrm{Au} / \mathrm{Ti} / \mathrm{TiO}_{2} / \mathrm{SiO}_{\mathrm{x}} / \mathrm{Si}-\mathrm{n}^{+}$devices the $\mathrm{ACF}$ and $\mathrm{PACF}$ are given in Figure 6. In this case, the influence of other lagged cycles is more important than in previous cases; therefore, the reset voltage can be given by a linear combination of reset voltages obtained in the previous cycles. The model is a factorized AR (6) type. 

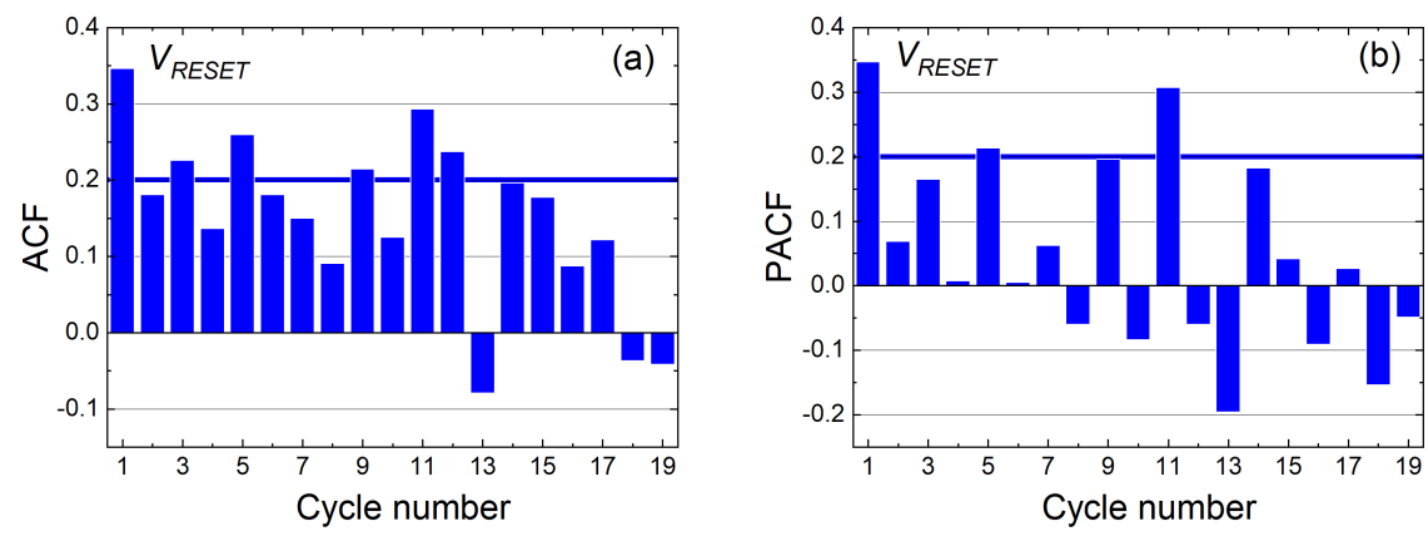

Figure 6. (a) ACF and (b) PACF versus cycle lag for the reset voltage of the $\mathrm{Au} / \mathrm{Ti} / \mathrm{TiO}_{2} / \mathrm{SiO}_{\mathrm{x}} / \mathrm{Si}-\mathrm{n}^{+}$devices under study and the $\mathrm{RS}$ series described in section II. The ACF and PACF minimum threshold bounds are 0.2 respectively, shown in blue lines.

The analytical expression for the TSSA description of the set voltage in $\mathrm{Au} / \mathrm{Ti} / \mathrm{TiO}_{2} / \mathrm{SiO}_{\mathbf{x}} / \mathrm{Si}-\mathrm{n}^{+}$devices is given by Equation 7.

$$
\begin{aligned}
V_{R E S E T_{t}}(V) & =0.3228+0.3198 V_{R E S E T_{t-1}}(V)+0.2197 V_{R E S E T_{t-5}}(V) \\
- & 0.0703 V_{R S E T_{t-6}}(V)
\end{aligned}
$$

The residuals of the $V_{R E S E T}$ models depicted in Equation 7 have white noise behavior again and consequently the modeling procedure is correct. Note that the coefficient of the (t-6) component is the product of the components (t-5) and ( $t-1)$ because of the characteristics of the parameter calculation procedure.

The three technologies under study here show stationarity in the set and reset voltages series. Nevertheless, for other technologies a drift in the mean and variance shows up. In these cases, stationarity does not hold, therefore stationarity is not a general rule. If we are faced with a nonstationary data series, the methodology described in the Supplementary Information would not be appropriate and no models can be extracted. In this situations there can be other options since the TSSA theory proposes 
changes of variables that lead the newly derived series to fulfill the stationary requirements that are needed prior to the modeling process. Autoregressive integrated moving average (ARIMA) approaches can be employed instead of the AR or ARMA modeling schemes explained above, $\operatorname{Ref}[30,31]$.

\section{5 - RESULTS AND DISCUSSION}

In order to test the accuracy of the models previously developed we superposed in the same graphic the measured $V_{S E T}$ and $V_{R E S E T}$ with the modeled ones for the devices under consideration in this manuscript. The modeling is a forecast of the actual value considering previous measured values, as it is conventionally done in TSSA. These results for $V_{S E T}$ (Figure 7) and $\mathrm{V}_{\text {RESET }}$ (Figure 8) are plotted taking into consideration $\mathrm{Ni} / \mathrm{HfO}_{2} / \mathrm{Si}-\mathrm{n}^{+}$and $\mathrm{Cu} / \mathrm{HfO}_{2} / \mathrm{Si}-\mathrm{n}^{+}$devices. As can be seen, the $V_{S E T}$ mean general trend is described reasonably well by Equations 2 and 3. The main dependencies have been correctly analyzed and incorporated with our procedure; hence, within the time series context, the model works well. We have validated this point by studying the residuals correlation, and we did not obtain any significant correlation between the residuals of the current cycle and those of the lagged ones (this constitutes the validation step, as explained in the Supplementary Information). Consequently, no more dependencies have to be incorporated to the models, since no information is statistically "hidden".

Although the scales and the modeling strategies are different for $V_{S E T}$, i.e. AR (1) for $\mathrm{Cu} / \mathrm{HfO}_{2} / \mathrm{Si}-\mathrm{n}^{+}$devices and $\operatorname{ARMA}(1,1)$ for $\mathrm{Ni} / \mathrm{HfO}_{2} / \mathrm{Si}-\mathrm{n}^{+}$devices, the accuracy is similarly reasonable, as Figure 7 shows. The model error $\left(\varepsilon_{t}\right)$, as highlighted before, presents a white noise structure. We have also checked that the model accuracy is maintained if the number interval is changed. 

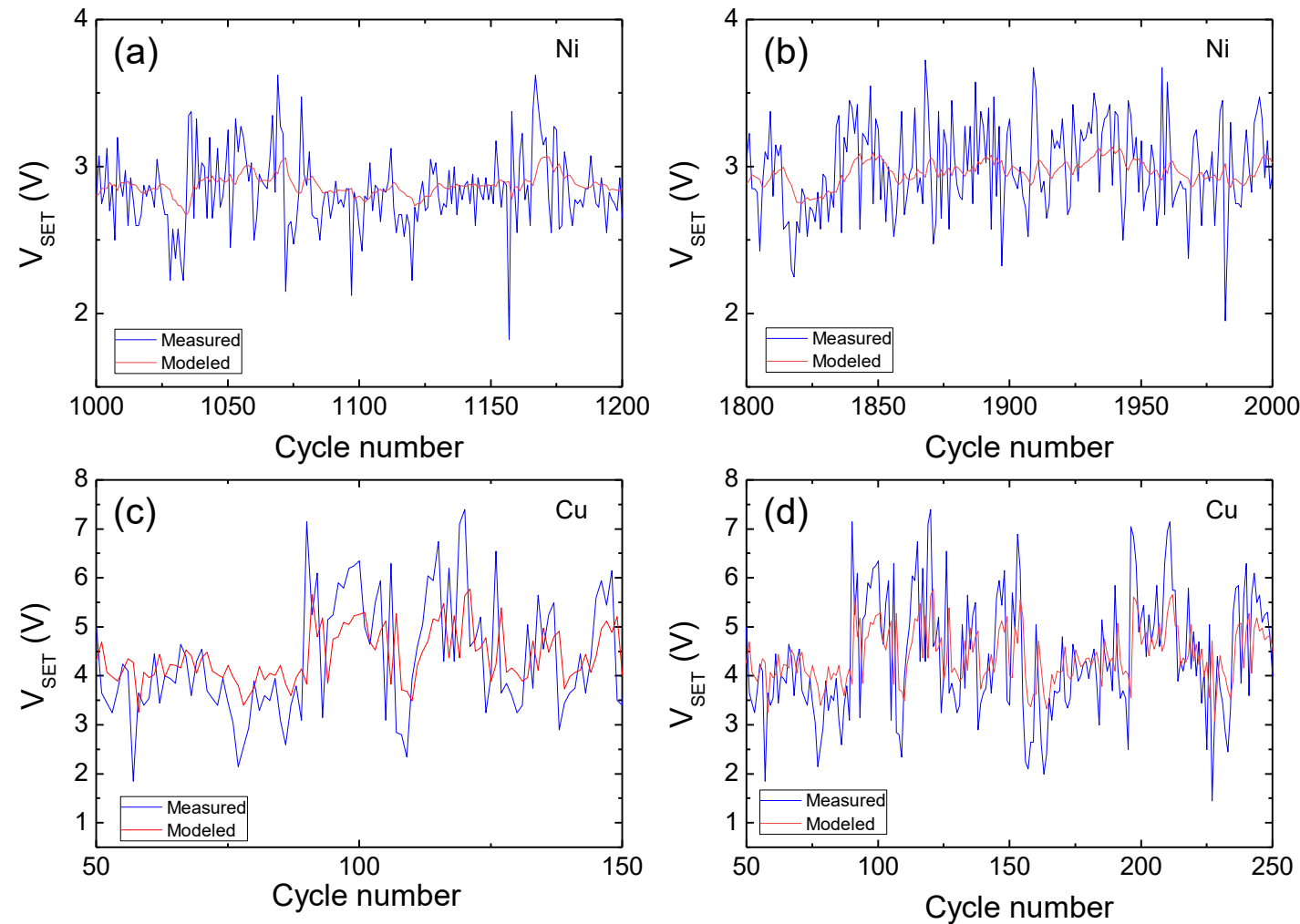

Figure 7. $V_{S E T}$ versus cycle number for the RS series under consideration. The measured values are shown in blue lines and the modelled ones in red. (a) $\mathrm{Ni} / \mathrm{HfO}_{2} / \mathrm{Si}^{-}$ $\mathrm{n}^{+}$RRAMs, cycles 1000-1200, (b) Ni/HfO $/ \mathrm{Si}^{+} \mathrm{n}^{+} \mathrm{RRAMs,} \mathrm{cycles} \mathrm{1800-2000} \mathrm{(c)}$ $\mathrm{Cu} / \mathrm{HfO}_{2} / \mathrm{Si}-\mathrm{n}^{+} \mathrm{RRAMs}$, cycles 50-150, (d) $\mathrm{Cu} / \mathrm{HfO}_{2} / \mathrm{Si}^{+} \mathrm{n}^{+} \mathrm{RRAMs}$, cycles 50-250.

The results for $V_{R E S E T}$ are in line with those of $V_{S E T}$. In this case, an AR (1) model is used for the $\mathrm{Cu} / \mathrm{HfO}_{2} / \mathrm{Si}-\mathrm{n}^{+}$devices and $\operatorname{ARMA}(1,1)$ for $\mathrm{Ni} / \mathrm{HfO}_{2} / \mathrm{Si}-\mathrm{n}^{+}$ devices. Again, the model reproduces accurately the $V_{R E S E T}$ mean evolution for all the cycle number intervals considered, as displayed in Figure 8. 

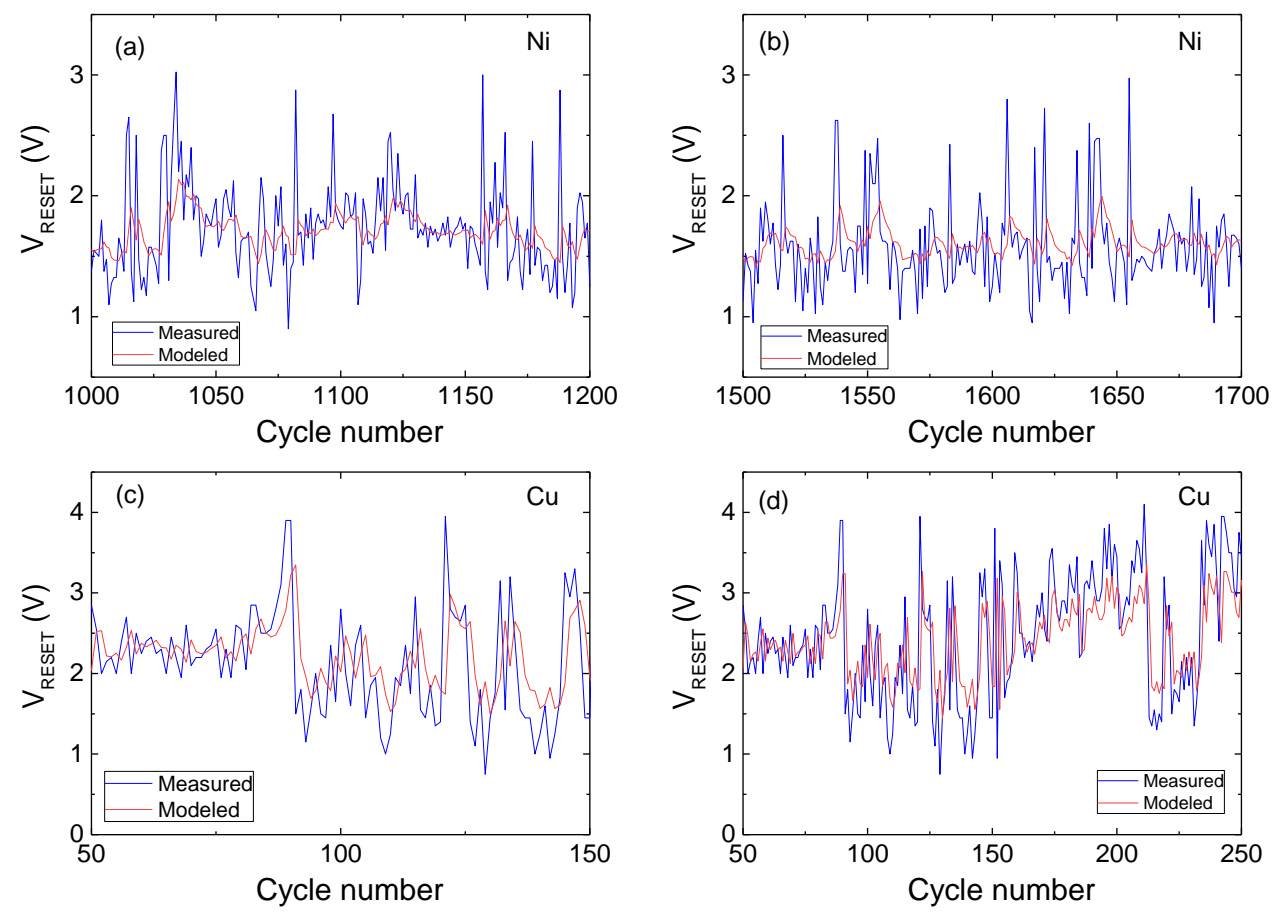

Figure 8. $V_{R E S E T}$ versus cycle number for the RS series under consideration. The measured values are shown in blue lines and the modeled ones in red. (a) $\mathrm{Ni} / \mathrm{HfO}_{2} / \mathrm{Si}^{-} \mathrm{n}^{+}$ RRAMs, cycles 1000-1200, (b) Ni/HfO $/ \mathrm{Si}^{+} \mathrm{n}^{+} \mathrm{RRAMs,} \mathrm{cycles} 1500-1700$ (c) $\mathrm{Cu} / \mathrm{HfO}_{2} / \mathrm{Si}-\mathrm{n}^{+} \mathrm{RRAMs}$, cycles 50-150, (d) $\mathrm{Cu} / \mathrm{HfO}_{2} / \mathrm{Si}-\mathrm{n}^{+} \mathrm{RRAMs}$, cycles 50-250.

For the $\mathrm{Au} / \mathrm{Ti} / \mathrm{TiO}_{2} / \mathrm{SiO}_{\mathrm{x}} / \mathrm{Si}-\mathrm{n}^{+}$devices the comparison between measured and modelled data is shown in Figure 9. The prediction is also reasonable although more values of set (Equation 4) and reset (Equation 7) voltages of previous cycles are considered. 

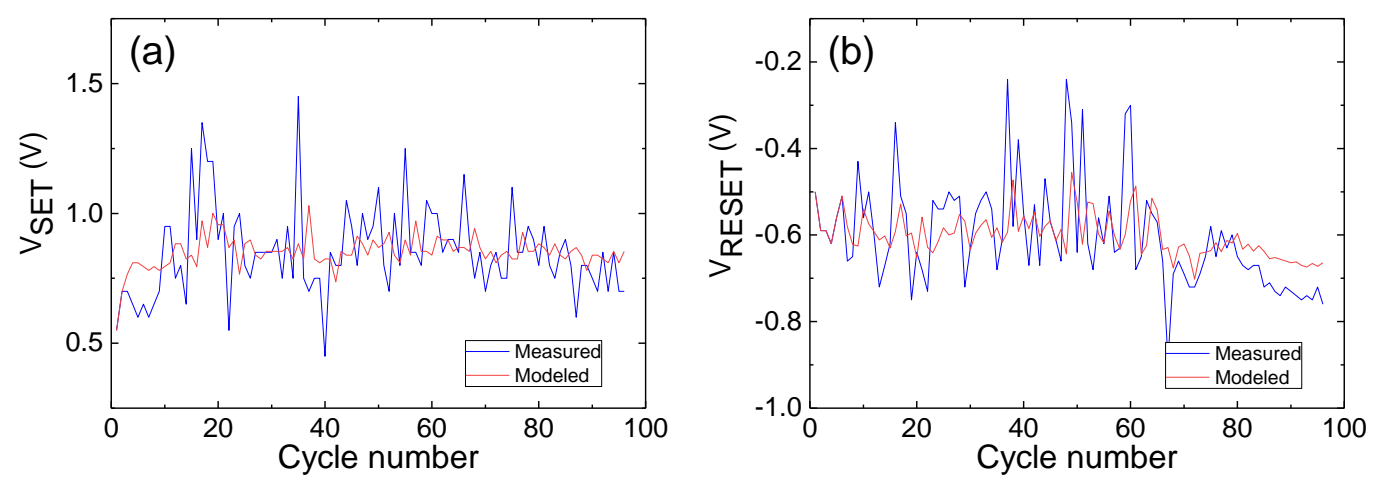

Figure 9. (a) Set (b) Reset voltage versus cycle number for the RS series under consideration. The measured values are shown in blue lines and the modeled ones in red. $\mathrm{Au} / \mathrm{Ti} / \mathrm{TiO}_{2} / \mathrm{SiO}_{\mathrm{x}} / \mathrm{Si}-\mathrm{n}^{+}$devices are considered here.

It can be observed in the figures above that in certain cases the fit is smaller than current values. This effect is seen when values much different than the mean show up. However, we would like to highlight that the modeling methodology we are presenting deals well with the prediction of the set and reset voltage mean. The current voltage values are also predicted well in most cases by using the statistical information of previous cycles. Therefore, taking into account that current models in the literature do not have this information, and that prediction of variability is interesting in devices (RRAMs) that show inherent stochasticity, we believe that, although it is not a modeling final solution, this technique is a step forward that can be worthwhile to characterize the device physics and help with variability modeling.

It is important to highlight that Figure 3 and Figure 5 (in addition to the data needed for model building) provide information about the RS processes of the $\mathrm{HfO}_{2}$ based devices analysed. In Figure 3a, we can see that the correlation between cycles is higher for the $\mathrm{Cu} / \mathrm{HfO}_{2} / \mathrm{Si}-\mathrm{n}^{+}$devices for the first lag cycles with respect to the set voltage. In Figure 5a, a similar trend can be observed for the reset voltage series. So, in general, the influence of previous cycles in $\mathrm{Cu} / \mathrm{HfO}_{2} / \mathrm{Si}-\mathrm{n}^{+}$devices is higher; i.e., the $\mathrm{CF}$ remnants from resets processes influence more the following set cycles, and that is why 
a higher correlation comes out for the set voltage values when several lag cycles are considered.

The qualitative explanation for this behaviour could be in the nature of RS in these devices. It is known that devices with $\mathrm{Cu}$ and $\mathrm{Ag}$ electrodes are employed in conductive-bridge RAMs [2, 36, 37] because of the capacity of $\mathrm{Cu}$ and $\mathrm{Ag}$ cations to diffuse in the dielectric and form, after a reduction process, a metallic-like conductive filament. In these devices, the reduction and oxidation potential are described by a thermally activated process whose activation energy depends on the number of atoms surrounding the one which is taken into consideration $[1,23,24]$. In this manner, the formation of the percolation path introduces "inertia" as the reduced atom clusters grow denser in the dielectric since they tend to maintain their shape hindering the oxidation processes of their atoms. This behaviour could explain the higher RS "inertia" shown by $\mathrm{Cu} / \mathrm{HfO}_{2} / \mathrm{Si}-\mathrm{n}^{+}$devices since they maintain better the $\mathrm{CF}$ form and size that determine $V_{S E T}$ and $V_{R E S E T}$ values. These effects would be reflected in the ACF plot with higher autocorrelation values for cycles not distant away in the series.

The lower autocorrelation (Figures $3 \mathrm{a}$ and $5 \mathrm{a}$ ) for the $\mathrm{Ni} / \mathrm{HfO}_{2} / \mathrm{Si}-\mathrm{n}^{+}$devices reflects a lower RS "inertia", as highlighted above. That could also be linked to a mixture of RS phenomena, since oxygen vacancies could be also involved in their resistive switching operation, as suggested in Ref. [38]. In this respect, the effects linked to activation energy lowering for oxidation of clustered metal atoms from the electrode would be mitigated, producing less correlation between the set and reset values of consecutive cycles.

In both cases, $\mathrm{Ni} / \mathrm{HfO}_{2} / \mathrm{Si}-\mathrm{n}^{+}$and $\mathrm{Cu} / \mathrm{HfO}_{2} / \mathrm{Si}-\mathrm{n}^{+}$devices, the most significant dependency (correlation) is with the previous value (first lag). In line with these latter issues, the results of $\mathrm{Au} / \mathrm{Ti} / \mathrm{TiO}_{2} / \mathrm{SiO}_{\mathrm{x}} / \mathrm{Si}-\mathrm{n}^{+}$devices can be analysed. In Figures 4 and 6 , 
the cycles correlated in the ACF and PACF (mostly for the reset voltage) is higher than for the technologies studied previously. In particular, for the reset voltage, the correlation of cycles $9,11,13$ is high with respect to the previous cases. This fact shows again an important inertia in the RS features of this technology in comparison to the devices based on $\mathrm{HfO}_{2}$. The nature of $\mathrm{CFs}$ in $\mathrm{TiO}_{2}$ for the filamentary current component and the presence of a volume current component (this component is linked to the ion distribution that is spread out in the dielectric and that can affect several consecutive cycles in a RS series, as it was shown in devices of this kind [29]), is key to explain the higher correlation highlighted above.

It is important to highlight that the autocorrelation and partial autocorrelation functions employed to in the analysis presented above (Figures 3-6) can be used separately from the TSSA modeling. They reflect the correlation of set and reset voltages between the different cycles, this means extracting the dependencies of the current cycle on the previous ones. This information is useful even if no TSSA modeling is performed since it shows that the values studied as independent data, are, in fact, dependent, and this fact has implications in the study of cycle-to-cycle variability. It is important to highlight that ACF and PACF analysis could be easily performed on RRAM measured data to assess the correlation between the characteristics of successive RS cycles.

From the modeling viewpoint, TSSA models could be implemented in circuit simulators with Verilog-A compilers. TSSA models could be embedded in previous models to account for the RRAM stochastic behavior and for the correlation of certain parameters such as set and reset voltages in long RS series, as explained above. We have done so making use of the RRAM Standford model [40]. In our case, a log file of previous set and reset values for the RRAMs has been employed in a model built upon the TSSA 
approach making use of regressed values. The file was written with the current values of set and reset voltages every time a set or reset was performed. Previously, in order to simulate the RVS case we described experimentally above, we implemented a modification of the local enhancement factor $(\gamma)$ [41] to be able to obtain the corresponding set and reset voltages, since these latter parameters are not model parameters.

Finally, we would like to comment on the fact that the operation regime of these devices would be characterized by pulses if they are used as storage-class memory in different chips or spikes of a variety of shapes if employed to mimic synapses in neuromorphic circuits. The device conductance depends on the pulse number for each signal amplitude, because of the different thermal inertia that is produced in each operation regime [39]. In this respect, we have chosen RVS measurements to show a particular application of the TSSA in a well-known characterization approach. This approach could be considered as the DC facet of a classical compact model, further developments could be needed to deal with transient events in a more general model where thermal and capacitive effects would be needed.

\section{VI.-CONCLUSIONS}

Times series statistical analyses (TSSA) have been used to study long series of resistive switching processes. The experimental data analyzed here were measured in resistive random access memories with $\mathrm{Ni} / \mathrm{HfO}_{2} / \mathrm{Si}-\mathrm{n}^{+}, \mathrm{Cu} / \mathrm{HfO}_{2} / \mathrm{Si}-\mathrm{n}^{+}$, $\mathrm{Au} / \mathrm{Ti} / \mathrm{TiO}_{2} / \mathrm{SiO}_{\mathrm{x}} / \mathrm{Si}-\mathrm{n}^{+}$structures. The conventional time series techniques were applied 
to model the $V_{S E T}$ and $V_{R E S E T}$ of these devices; to do so, autocorrelation functions and partial autocorrelation functions were obtained for all the types of RRAMs. Autoregressive models were obtained for $\mathrm{Cu} / \mathrm{HfO}_{2} / \mathrm{Si}-\mathrm{n}^{+}$devices and the autocorrelation function between cycles was high, showing an important inertia between resistive switching cycles. The better diffusion of $\mathrm{Cu}$ ions in the dielectric is behind this behaviour. For $\mathrm{Ni} / \mathrm{HfO}_{2} / \mathrm{Si}-\mathrm{n}^{+}$devices more complex models are needed and autocorrelation data show less inertia between resistive switching cycles. Finally, for $\mathrm{Au} / \mathrm{Ti} / \mathrm{TiO}_{2} / \mathrm{SiO}_{\mathrm{x}} / \mathrm{Si}-\mathrm{n}^{+}$devices a significant correlation can be observed for more distant cycles in the reset voltage description. A physical explanation has been developed in connection with the correlation results found. The models obtained can be used to forecast the values of set and reset voltages in a resistive switching series if previous values are known. The information obtained in this context can be employed in modelling and in the characterization of RRAM variability. 


\section{Supplementary Information}

In this paper, Box-Jenkins methodology [31] is applied for modeling the time series of reset and set voltages in resistive RRAMs. The formulation of an ARMA(p,q) model is given as follows (the considerations would be the same for the set voltage),

$$
V_{R E S E T_{t}}=\Phi_{0}+\Phi_{1} V_{R E S E T_{t-1}}+\cdots+\Phi_{p} V_{R E S E T_{t-p}}-\theta_{1} \varepsilon_{t-1}+\cdots-\theta_{q} \varepsilon_{t-q}
$$

where $V_{\text {RESET }_{t}}$ is the modeled reset voltage in the current cycle of an RS series, and $\mathrm{VESET}_{\mathrm{t}-\mathrm{k}}$ are the modelled reset voltages lagged $\mathrm{k}$ cycles (i.e., the $\mathrm{k}$ reset voltage values of the previous RS cycles), $\varepsilon_{\mathrm{t}-\mathrm{k}}$ are the errors (residuals) made in the modeling process from earlier cycles, with $\Phi_{i}(i=1, \ldots, p)$ and $\theta_{j}(j=1, \ldots, q)$ being the unknown regression coefficients to be estimated in the modelling process. In time series methodology the term $\varepsilon_{t}$ is also included in the model but, in our case, we assume it. A similar description holds for the set voltage.

The current value of the modeled reset voltage can be calculated by means of two linear polynomials, one for the autoregressive part (AR, in this case the reset voltage is modeled as a linear function of some of its past values), and the other for the moving average part (MA, a linear combination of the past model errors or residuals). The parameters $\mathrm{p}$ and $\mathrm{q}$ are the orders of the autoregressive part and the moving average part, respectively.

The ARMA approach assumes that the time series is stationary and the model error $\varepsilon_{\mathrm{t}}$ shows white noise behaviour (uncorrelated random errors). The ARMA 
modeling process can be performed in five main steps: checking stationarity, model identification, parameter estimation, validation and prediction.

\section{ACKNOWLEDGMENTS}

We would like to thank F. Campabadal and M. B. González from the IMB-CNM (CSIC) in Barcelona for fabricating and providing the experimental measurements of the devices employed here. The authors thank the support of the Spanish Ministry of Science, Innovation and Universities under projects TEC2017-84321-C4-3-R and MTM2017-88708-P (also supported by the FEDER program). This work has made use of the Spanish ICTS Network MICRONANOFABS. 


\section{References}

[1] F. Pan, S. Gao, C. Chen, C. Song, F. Zeng, "Recent progress in resistive random access memories: materials, switching mechanisms and performance", Materials Science and Engineering, 83, pp. 1-59, 2014.

[2] D. Ielmini, R. Waser. "Resistive Switching: From Fundamentals of Nanoionic Redox Processes to Memristive Device Applications”, Wiley-VCH, 2015.

[3] R. Waser and M. Aono, "Nanoionics-based resistive switching", Nature materials, 6, pp. 833-840, 2007.

[4] M. Lanza, G. Bersuker, M. Porti, E. Miranda, M. Nafría, X. Aymerich, "Resistive switching in hafnium dioxide layers: Local phenomenon at grain boundaries", Applied Physics Letters, vol. 101, 193502, 2012.

[5] M. Lanza, H.-S. P. Wong, E. Pop, D. Ielmini, D. Strukov, B.C. Regan, L. Larcher, M.A. Villena, J.J. Yang, L. Goux, A. Belmonte, Y. Yang, F. M. Puglisi, J. Kang, B. Magyari-Köpe, E. Yalon, A. Kenyon, M. Buckwell, A. Mehonic, A. Shluger, H. Li, T.-H. Hou, B. Hudec, D. Akinwande, R. Ge, S. Ambrogio, J.B. Roldan, E. Miranda, J. Suñe, K.L. Pey, X. Wu, N. Raghavan, E. Wu, W.D. Lu, G. Navarro, W. Zhang, H. Wu, R. Li, A. Holleitner, U. Wurstbauer, M. Lemme, M. Liu, S. Long, Q. Liu, H. Lv, A. Padovani, P. Pavan, I. Valov, X. Jing, T. Han, K. Zhu, S. Chen, F. Hui, Y. Shi, "Recommended methods to study resistive switching devices", Advanced Electronics Materials, 2018, 1800143 (DOI: 10.1002/aelm.201800143).

[6] R. Waser (ed.), "Nanoelectronics and Information Technology", 3rd edition, Wiley, 2012. 
[7] M.A. Villena, J.B. Roldán, F. Jiménez-Molinos, E. Miranda, J. Suñé, M. Lanza, "SIM"RRAM: A physical model for RRAM devices simulation", Journal of Computational Electronics, in press, 2017.

[8] R. Degraeve, A. Fantini, N. Raghavan, L. Goux, S. Clima, B. Govoreanu, A. Belmonte, D. Linten, M. Jurczak, "Causes and consequences of the stochastic aspect of filamentary RRAM”, Microelectronic Engineering, 147, pp. 171-175, 2015.

[9] F.M. Puglisi, N. Zagni, L. Larcher, P. Pavan, "Random Telegraph Noise in Resistive Random Access Memories: Compact Modeling and Advanced Circuit Design”, IEEE Transactions on Electron Devices, 65, pp. 2964 - 2972, 2018.

[10] S. Menzel, P. Kaupmann, R. Waser, "Understanding filamentary growth in electrochemical metallization memory cells using kinetic Monte Carlo simulations”, Nanoscale, 7, 12673, 2015.

[11] S. Long, X. Lian, T. Ye, C. Cagli, L. Perniola, E. Miranda, M. Liu, and J. Suñé, "Cycle-to-cycle intrinsic RESET statistics in $\mathrm{HfO}_{2}$-based unipolar RRAM devices”, IEEE Electron Device Lett. 34(5), 623-625, 2013.

[12] W.C, Luo, J.C. Liu, H.T. Feng, Y.C. Lin, J.J. Huang, K.L. Lin, T.H. Hou, "RRAM SET speed-disturb dilemma and rapid statistical prediction methodololy", International Electron Device Meeting, p. 9.5.1, 2012.

[13] J.W. McPherson, "Reliability Physics and Engineering. Time-to-Failure Modeling”, second ed. Springer, 2013. 
[14] E. Y. Wu, B. Li, J.H. Stathis, "Modeling of time-dependent non-uniform dielectric breakdown using a clustering statistical approach", Applied Physics Letters, 103, p. 152907, 2013.

[15] E. Y. Wu, E. J. Nowak, R. Vollertsen, and L. K. Han, "Weibull Breakdown Characteristics and Oxide Thickness Uniformity", IEEE Trans. Electron Device 47, 2301-2309, 2000.

[16] C. Acal, J.E. Ruiz-Castro, A. M. Aguilera, F. Jiménez-Molinos, J.B. Roldán, "Phase-type distributions for studying variability in resistive memories", Journal of Computational and Applied Mathematics, 345, pp. 23-32, 2019.

[17] H. Tian, X.-F. Wang, M. A. Mohammad, G.-Y. Gou, F. Wu, Y. Yang, T.-L. Ren, "A hardware Markov chain algorithm realized in a single device for machine learning", Nature communications, 9, 4305, 2018.

[18] F.M. Puglisi, P. Pavan, "RTN analysis with FHMM as a tool for multi-trap characterization in $\mathrm{HfO}_{\mathrm{X}}$ RRAM”, 2013 IEEE International Conference of Electron Devices and Solid-state Circuits, DOI: 10.1109/EDSSC.2013.6628059

[19] F. Pan, S. Yin, V. Subramanian, "A detailed study of the forming stage of an electrochemical resistive switching memory by KMC simulation," IEEE Electron Device Lett. 32, 949-951, 2011.

[20] X. Guan, Member, IEEE, S. Yu, H.S. Philip Wong, "On the switching parameter variation of metal-oxide RRAM-Part I: Physical modeling and simulation methodology", IEEE Transactions on Electron Devices, 59, pp. 1172-1182, 2012.

[21] L. Larcher, A. Padovani, O. Pirrotta, L. Vandelli, and G. Bersuker, "Microscopic understanding and modeling of HfO2 RRAM device physics," in Proc. IEEE Int. Electron Devices Meeting, pp. 20.1.1-20.1.4, 2012. 
[22] J. Guy, G. Molas, P. Blaise, M. Bernard, A. Roule, G. Le Carval, V. Delaye, A. Toffoli, G. Ghibaudo, Fellow, IEEE, F. Clermidy, B. De Salvo, L. Perniola, "Investigation of Forming, SET, and Data Retention of Conductive-Bridge RandomAccess Memory for Stack Optimization", IEEE Transactions on Electron Devices, 62(11), pp. 3482-3489, 2015

[23] S. Aldana, P. García-Fernández, A. Rodríguez-Fernández, R. RomeroZaliz, M.B. González, F. Jiménez-Molinos, F. Campabadal, F. Gómez-Campos, J.B. Roldán, "A 3D Kinetic Monte Carlo simulation study of Resistive Switching processes in Ni/HfO2/Si-n+-basedRRAMs", Journal of Physics D: Applied Physics, 50, 335103, 2017.

[24] S. Aldana, J.B. Roldán, P. García-Fernández, J. Suñe, R. Romero-Zaliz, F. Jiménez-Molinos, S. Long, F. Gómez-Campos, M. Liu, “An in-depth description of bipolar resistive switching in $\mathrm{Cu} / \mathrm{HfOx} / \mathrm{Pt}$ devices, a 3D Kinetic Monte Carlo simulation approach”, Journal of Applied Physics, 123, 154501, 2018.

[25] M. Schie, S. Menzel, J. Robertson, R. Waser and R. A. De Souza, "Fieldenhanced route to generating anti-Frenkel pairs in HfO2", Physical Review Materials, 2, 035002, 2018.

[26] M. Xie, S.L. Ho, "Analysis of repairable system failure data using time series models", Journal of Quality in Maintenance Engineering, Vol. 5 Issue: 1, pp.50$61,1999$.

[27] K. L. Lee \& S. A. Billings, "Time series prediction using support vector machines, the orthogonal and the regularized orthogonal least-squares algorithms", International Journal of Systems Science, 33:10, 811-821, DOI: 10.1080/0020772021000017317, 2002. 
[28] M. B. González, J. M. Rafí, O. Beldarrain, M. Zabala, and F. Campabadal, "Analysis of the switching variability in Ni/HfO2-based RRAM devices," IEEE Trans. Device Mater. Reliab., vol. 14, no. 2, pp. 769-771, 2014.

[29] N. Xiao, M.A. Villena, B. Yuan, S. Chen, B. Wang, M. Elias, Y. Shi, F. Hui, X. Jing, A. Sheuermann, K. Tang, P.C. McIntyre, M. Lanza, "Resistive Random Access Memory Cells with a Bilayer $\mathrm{TiO}_{2} / \mathrm{SiO}_{X}$ Insulating Stack for Simultaneous Filamentary and Distributed Resistive Switching", Advanced Functional Materials, 27, p. $1700384,2017$.

[30] P. J. Brockwell, R. A. Davis, "Introduction to Time Series and Forecasting”, Second Edition, Springer, 2002.

[31] S.Bisgaard, M.Kulahci, "Time series analysis and forecasting by example", Wiley, 2011.

[32] G.U. Yule, "On a method of investigating periodicities in disturbed series, withreference to Wolfer's Sunspot Numbers", Philosophical Transactions of the Royal Society of London, Series A, 226, pp. 267-298, 1927.

[33] D. C. Montgomery, C. L. Jennings, M. Kulahci, "Introduction to Time Series Analysis and Forecasting", Wiley Series in Probability and Statistics, Wiley, 2015.

[34] M.A. Villena, J.B. Roldán, F. Jiménez-Molinos, J. Suñé, S. Long, E. Miranda, M. Liu, "A comprehensive analysis on progressive reset transitions in RRAMs", Journal of Physics D: applied physics, 7, 205102, 2014.

[35] A. Coghlan, "A little book of $\mathrm{R}$ for time series", Release 0.2. In: https://media.readthedocs.org/pdf/a-little-book-of-r-for-time-series/latest/a-little-bookof-r-for-time-series.pdf, 2018. 
[36] Yang, Y. et al. Electrochemical dynamics of nanoscale metallic inclusions in dielectrics. Nat. Commun. 5, 4232, 2014.

[37] Yuanyuan Shi, Xianhu Liang, Bin Yuan, Victoria Chen, Haitong Li, Fei Hui, Zhouchangwan Yu, Fang Yuan, Eric Pop, H.-S. Philip Wong, Mario Lanza ,"Electronic synapses made of layered two-dimensional materials“", Nature Electronics $1,458-465,2018$.

[38] U. Celano, L.Goux, R. Degraeve, A.Fantini, O. Richard, H. Bender, M.Jurczak, W.Vandervorst, "Three-Dimensional Observation of the Conductive Filament in Nanoscaled Resistive Memory Devices", Nanoletters, 14, pp. 2401-2406, 2014

[39] S. Kim, C. Du, P. Sheridan, W. Ma, S. Choi, W. D. Lu, "Experimental Demonstration of a Second-Order Memristor and Its Ability to Biorealistically Implement Synaptic Plasticity", NanoLetters, 15, 2203, 2015.

[40] https://nano.stanford.edu/stanford-rram-model

[41] X. Guan, S. Yu, and H-S.P Wong, “A SPICE Compact Model of Metal Oxide Resistive Switching Memory with Variations”, IEEE Elec. Dev. Lett., vol. 33, pp. 1405-1407, 2012. 